\title{
Effects of stimulus features and instruction on response coding, selection, and inhibition: Evidence from repetition effects under task switching
}

\author{
Michel D. Druey and Ronald Hübner \\ Universität Konstanz, Konstanz, Germany
}

\begin{abstract}
The coding of stimuli and responses is crucial for human behaviour. Here, we focused primarily on the response codes (or response categories). As a method, we applied a combined dual-task and taskswitch paradigm with a fixed task-to-hand mapping. Usually, negative effects (i.e., costs) are observed for response category repetitions under task switching. However, in several previous studies it has been proposed that such repetition effects do not occur, if the stimulus categories (e.g., "odd" if digits have to be classified according to their parity feature) are unequivocally mapped to specific responses. Our aim was to test this hypothesis. In the present experiments, we were able to distinguish between three different types of possible response codes. The results show that the participants generally code their responses according to abstract response features (left/right, or index/middle finger). Moreover, the spatial codes were preferred over the finger-type codes even if the instructions stressed the latter. This preference, though, seemed to result from a stimulus-response feature overlap, so that the spatial response categories were primed by the respective stimulus features. If there was no such overlap, the instructions determined which type of response code was involved in response selection and inhibition.
\end{abstract}

Keywords: Repetition effects; Response coding; Task switching; Attention.

One of the central questions in psychology concerns how perception and action are related. It is generally assumed that, by instruction and practice, people learn to respond to stimuli according to a required task. Several models have been proposed in order to explain how the respective stimulus-response (SR) associations are built up and how they are retrieved from memory (see, e.g., Botvinick, Nystrom, Fissell, Carter, \& Cohen, 1999; Cohen \& Shoup, 1997; Hommel, Müsseler, Aschersleben, \& Prinz, 2001; Logan, 1988, 2002; Meiran, 2000a; Pashler \& Baylis, 1991; Zhang, Zhang, \& Kornblum, 1999). A crucial issue in this respect concerns the involved mental stimulus and response codes, since it is obvious that the

Correspondence should be addressed to Michel D. Druey, Universität Konstanz, Fachbereich Psychologie, Fach D29, D-78457 Konstanz, Germany. E-mail: michel.druey@uni-konstanz.de

We thank Thomas Kleinsorge, Iring Koch, Nachshon Meiran, Mike Wendt, Marco Steinhauser, and Tobias Studer for their helpful comments on earlier versions of this manuscript. Furthermore, we are grateful to Cindy Eckart, Johannes Schult, and Karen Donath for collecting the data. This research was supported by a grant (Hu 432/9) from the Deutsche Forschungsgemeinschaft (DFG) to the second author. 
stimuli and the responses are not directly connected, but rather via a more or less complex conceptual structure (i.e., stimulus and response categories).

In the present study, we applied a combined dual-task and task-switch paradigm (cf. e.g., Hübner \& Druey, 2006) in order to investigate primarily those mental representations that are relevant for response selection. In order to achieve this goal, we specifically focused on the effects of response category repetitions. However, before we report our design and our results in detail, some of the relevant literature on stimulus and response categories is considered.

\section{Stimulus and response categories}

Visual objects can be described by their basic physical features, such as their colour, size, and so on (cf. Treisman, 1998). Furthermore, many objects also have abstract features. For instance, numerals can be odd or even, or letters can be vowels or consonants. Basic as well as abstract features can be used for the categorization of objects or stimuli. In various experimental paradigms, this is utilized to construct simple mental tasks, which serve for the investigation of human performance. Models in this field are usually based on the assumption that stimulus categories are mentally represented units, which are activated by corresponding stimulus features. If a unit, or a combination of units, exceeds a certain threshold, then the stimulus is categorized accordingly (e.g., Houghton \& Tipper, 1994; Lamberts, 2000; Nosofsky \& Palmeri, 1997).

However, in order to observe the resulting categorization, individuals also have to respond in some way. Thus, besides categorizing the stimuli with respect to one or more features, the participants also have to indicate the result of this categorization by pressing one of several buttons according to a prespecified SR mapping (e.g., Campbell \& Proctor, 1993; Hommel, 1998a; Pashler \& Baylis, 1991). In order to define these mapping rules, the responses are defined in one or the other way. Thus, not only the stimuli but also the responses have features, which are mentally represented in terms of response categories (e.g., Kornblum, Hasbroucq, \& Osman, 1990). From a large number of studies, which are often concerned with stimulus-response compatibility (SRC) effects, it is well known that both stimulus and response features and their respective mental representations play a crucial role with respect to performing simple reactive tasks (e.g., Hedge \& Marsh, 1975; Heister, Schroeder-Heister, \& Ehrenstein, 1990; Hommel, 1997; Hommel et al., 2001; Kornblum et al., 1990; Kornblum \& Lee, 1995; Lu \& Proctor, 1995; Wallace, 1971). This is most evident in studies applying the Simon task (Simon, 1969). In this task, participants have to categorize stimuli with respect to some nonspatial features such as colour (e.g., Hedge \& Marsh, 1975) or form (e.g., Wallace, 1971), and individual feature values (e.g., red or circle) are assigned to a left or right response key, respectively. The crucial point is that the stimuli are presented laterally even though stimulus position is task irrelevant. As a result, participants respond faster to stimuli appearing at the same side as the required response than to stimuli presented at the opposite side. In other words, there is an advantage if the irrelevant spatial feature of the stimulus corresponds to the spatial feature of the response. This advantage, which is commonly referred to as the Simon effect, shows that (a) responses are also coded according to their spatial features, and (b) there might exist a dimensional overlap between stimulus and response codes (cf. Kornblum et al., 1990).

In some Simon task studies, it has furthermore been shown that different frames of reference are possible with respect to response coding. This means that responses can be coded according to different features. Hommel (1993), for instance, used a Simon task, in which a left or right light was switched on by pressing a right or left button, respectively. By instruction, the participants were encouraged to code their responses either in terms of the spatial position of the response keys or in terms of the position of the appearing light. It turned out that, depending on which instruction the participants received, the respective Simon effects were in opposite 
directions. This shows that the participants followed the instructions and either used key position or light position as response codes.

Similarly, Proctor, Wang, and Pick (2004; see also Stins \& Michaels, 1997) asked their participants to hold a steering wheel with both hands either at the top or at the bottom position and to respond to high- or low-pitch tones, which were presented either to the left or to the right ear, with clockwise or counterclockwise wheel rotations. At the top position, wheel rotation (e.g., clockwise, i.e., steering to the right) and hand movement (e.g., to the right) were spatially congruent. Accordingly, the usual SRC (Simon) effect occurred. However, at the bottom position, wheel rotation (e.g., steering to the right) and hand movement (e.g., to the left) were opposite. Under this condition, a negative $\mathrm{SRC}$ effect occurred, but only if the participants were instructed according to hand movement. Otherwise, no SRC effect occurred. In an earlier study using a similar task, Stins and Michaels (1997) showed that, with the hand at the bottom position and neutral instruction, some participants coded their responses according to the wheel rotation, whereas others used hand movement direction as response code.

Together, these results show that (a) responses can be coded by different spatial features, and (b) the instruction plays a crucial role as to which of these features is used for response coding. Furthermore, without specific instruction, different participants may use different codes.

\section{Response category repetitions}

Besides SR feature overlap, also overlapping features between different responses may tell us something about response coding and selection. However, as in most of the SRC studies, in corresponding studies the focus was almost exclusively on spatial (primarily left/right) response features (e.g., Hazeltine, 2005; Hommel, 1998a; Hübner \& Druey, 2006; Lien \& Proctor, 2000; Lien, Schweickert, \& Proctor, 2003; Schuch \& Koch, 2004). Only a few studies have nonspatial features such as finger type (e.g., Campbell \& Proctor, 1993; Watter \& Logan, 2006) or colour (e.g.,
Wenke \& Frensch, 2005) have also been considered.

To illustrate how questions concerning response coding and selection can be answered by analysing response correspondence and response repetition effects, we consider the study of Campbell and Proctor (1993) in more detail. They had participants judge single stimuli according to whether they were letters, digits, or symbols. Each of these stimulus categories was mapped to an individual button on each of two response boxes, which had to be pushed by corresponding fingers (i.e., index, middle, and ring fingers) of both hands. Most importantly, responding alternated between the left and right hand across trials. Thus, by varying the arrangement of the response keys, the effects of repeating different "salient features" (Campbell \& Proctor, 1993) of the responses could be investigated.

In one condition (Experiment 5, see Figure 1 right panel), for instance, the response keys for the left and right hand were arranged horizontally and vertically, respectively, and the stimulus categories were mapped to individual finger types. This resulted in consistent (or invariant, see below) SR mappings according to the anatomical finger features for both hands. From the resulting benefits for finger-type repetitions relative to finger-type switches across trials, Campbell and Proctor (1993) concluded that the responses were coded according to the finger-type features.

In another condition (Experiment 4, see Figure 1 middle panel), the buttons on both boxes were arranged horizontally, and the stimulus categories were mapped to the spatial features of the respective response keys (i.e., left, middle, and right response keys). Since Campbell and Proctor (1993) observed benefits for the repetition of these spatial features from one trial to the next, they concluded that now the participants used left/middle/right as response codes. This is interesting, since the keys still had to be pressed with the index, middle, and ring fingers. Thus, with the horizontal key arrangement, it would also have been possible for the participants to code the responses according to the finger type used for responding. This raises the question of why the participants used the spatial 


\section{Experiment 2}

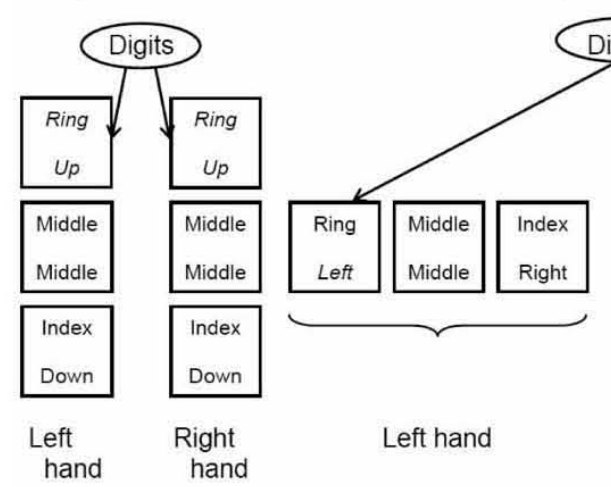

Experiment 4

Figure 1. Schematic depiction of the stimulus category to response category mapping (for the digit stimulus category) in Experiments 2, 4, and 5 in the study of Campbell and Proctor (1993). In the boxes, the upper line always indicates the possible response categories if the responses are coded according to the finger type used for responding, the lower line always indicates the possible response categories if the responses are coded according to the spatial features of the response buttons. Those response categories that were used for response selection (as concluded from the respective repetition effects) are written in italic.

and not the anatomical response features as response codes. To answer this question, it is important to note that the spatial and the finger-type codes do not correspond across hands. Whereas, for instance, the index finger of the left hand pushes the rightmost button, the index finger of the right hand pushes the leftmost button. Thus, if the spatial category repeats from one hand to the other, the finger category changes, and vice versa. We return to this issue below.

First, though, let us consider the situation in which the response buttons for the two hands were both arranged vertically (Campbell \& Proctor, 1993, Exp. 2). As can be seen in Figure 1 (left panel), pressing the buttons at the bottom of the response boxes required the index fingers of both hands. That is, with such an arrangement, the finger and the spatial mappings are consistent/invariant across hands. As expected, corresponding responses (according to the spatial and finger-type response features) led to faster responses from one trial to the next. However, contrary to the other conditions with orthogonal and horizontal key arrangements for the two hands, it is unclear which response features were used for response coding under this condition.
Given these facts, one may ask (a) why responses produced with different effectors are related through abstract categories at all, and (b) which factors determine the category type actually involved in response selection (e.g., in the situation with the vertically arranged response keys in the second experiment of Campbell \& Proctor, 1993). A possible answer to these questions could be that common categories are helpful, especially at the beginning of an experiment, when the SR mapping has still to be learned. Common features can then be used to facilitate the retrieval of the individual SR relations from memory. For instance, if a certain stimulus category is mapped to the leftmost response button for each hand, then this relation can be learned and remembered easier than that the category is mapped to the middle finger of the left hand and to the index finger of the right hand. Thus, participants might preferentially represent their responses by categories that remain invariant across hands (cf. Duncan, 1979). According to Campbell and Proctor (1993), this salient-features coding principle (as they termed it) also explains why the spatial and not the anatomical response features were used for coding the responses with 
horizontally arranged response keys for both hands (Figure 1, middle panel).

However, invariance relations seem to be crucial primarily in simple choice task conditions - that is, if the task repeats. Under such conditions, response repetition benefits are usually observed (e.g., Campbell \& Proctor, 1993; Hübner \& Druey, 2006; Schuch \& Koch, 2004). Under task switch conditions, though, such invariance relations are usually not given a priori. Nevertheless, even under such conditions, response category repetition effects have been observed (Hübner \& Druey, 2006; Schuch \& Koch, 2004), but in this case costs instead of benefits occurred (see also Kleinsorge, 1999; Mayr \& Klieg1, 2003; Meiran, 2000a; Rogers \& Monsell, 1995, for similar effects with physical response repetitions).

Although several accounts have been proposed to explain this interaction between task switching and response category repetition (cf. Rogers \& Monsell, 1995), recent data support the idea that there is a general tendency to inhibit the previously relevant response category (Hübner \& Druey, 2006; Steinhauser \& Hübner, 2006). Furthermore, this inhibition seems to be targeted exclusively to those response categories, according to which the SR mapping is defined and on which the intentional SR translation is based, even if other categories are also involved and are activated as well (Hübner \& Druey, in press). The result that there is a repetition benefit on task repetition trials can be explained by the assumption that on these trials the stimulus category also repeats, which outweighs the costs of response inhibition.

Although questions concerning the origin of the different repetition effects are of great interest, they were not in the main focus of the present study. Rather, these effects served as a tool for investigating the mechanisms of response coding. That response category repetition effects have also been observed under task switching shows that abstract response categories play a crucial role for response selection also under these conditions. However, in view of the nonexistent direct invariance relations, how can the respective effects be explained?

At least two accounts seem to be possible. First, abstract response coding under task switching could be induced by the requirement to also perform task repetition trials. In our previous study (Hübner \& Druey, 2006), for instance, the participants had to perform task switches as well as task repetitions. Thus, the existing spatial invariance relations between the two hands under task repetition may have generalized to the task switch blocks, presumably since the same stimuli and tasks were relevant in both conditions. In other words, stimulus category-response category associations were established, which then could be used also in the task switch blocks. Consequently, the same abstract response codes were used under task switching as under task repetition. However, this explanation does not hold for the study of Schuch and Koch (2004), since their participants performed either only task repetition blocks or task switch blocks.

Therefore, another explanation is required to explain why Schuch and Koch (2004) observed repetition effects under task switch conditions. In their study they applied a dual-task paradigm, in which the participants performed both judgement types (parity and magnitude judgements of numerals) as Task $1\left(\mathrm{~T}_{1}\right)$ and Task $2\left(\mathrm{~T}_{2}\right.$; see also Hübner \& Druey, 2006). As a consequence, within-trial task order changed from one trial to the next (e.g., parity as $T_{1}$ and magnitude as $T_{2}$ on trial $n-1$; magnitude as $\mathrm{T}_{1}$ and parity as $\mathrm{T}_{2}$ on trial $n$ ). In other words, both judgement types had to be performed with both hands (i.e., both response alternatives on each hand were associated with both judgement types). This presumably established invariance relations within each hand rather than (or additionally to) between hands, ${ }^{1}$ since the mapping of the stimulus

\footnotetext{
${ }^{1}$ The participants in the study of Schuch and Koch (2004) actually responded verbally ("left", "right") to the first stimulus ( $\left.\mathrm{S}_{1}\right)$ and manually (left and right response buttons) to the second stimulus $\left(\mathrm{S}_{2}\right)$. Notice, however, that this neither questions the reasoning presented here, since it holds for $\mathrm{R}_{2}$ (the manual response), which is relevant with respect to the repetition effects, nor precludes that the present logic can be applied to a situation with only manual responses for $T_{1}$ and $T_{2}$.
} 
to the respective spatial (left/right) response categories was identical for both hands.

Notice, though, that for both of these explanations a critical issue concerns the fact that the responses for both tasks are related to both hands (cf. Lien \& Proctor, 2002). Whether a variable task-to-hand mapping is indeed necessary for abstract response coding and, consequently, for repetition effects to occur, is one of the central questions under investigation in the present study.

\section{The present experiments}

In the present study, we applied a combined PRP (psychological refractory period) and task switch paradigm (cf. Hübner \& Druey, 2006). However, contrary to our previous studies (Hübner \& Druey, 2006, in press) and to the study of Schuch and Koch (2004), the task order and the task-to-hand mapping remained constant for each participant throughout the whole experiment. Since we also excluded task repetitions, invariant SR mappings could be established neither between nor within hands for any specific response category type (see Figure 2 for an illustration).

The participants had to respond to the first stimulus $\left(\mathrm{S}_{1}\right)$ and to the second stimulus $\left(\mathrm{S}_{2}\right)$ on each trial by pressing a left or right response button with the index and middle fingers of their left and right hands, respectively. Figure 2 exemplarily shows the SR mappings for the two tasks primarily applied in the present study. As can be seen, the responses can be coded in terms of at least three different features. First, they can be coded according to individual features for each response (e.g., the individual effectors, see Figure 2). Besides the fact that this would be optimal for avoiding response conflict, it is also what is assumed in several classification models, which were proposed in order to explain the results from a wide range of PRP and task switch studies (e.g., Gilbert \& Shallice, 2002;
Logan \& Gordon, 2001; Logan \& Schulkind, 2000; Meiran, 2000a, 2000b). Second, the responses can also be coded in terms of response features. In this case the anatomical features of the effectors (i.e., finger type), ${ }^{2}$ can be used, but also the spatial features of the response buttons (i.e., left/right) are possible.

Which one of these possible response category types is actually involved in response selection and inhibition should be discernible by the respective repetition effects. Two results are indicative in this respect. First, whether the responses are coded according to the individual effectors or abstract response features is indicated by the absence or presence of response category repetition effects, respectively. Second, contrary to the study of Campbell and Proctor (1993, Exp. 2), the present procedure also allows one to differentiate between the two possible types of abstract response codes (i.e., index/middle finger versus left/right button). Since response category repetitions usually result in costs under task switching (e.g., Hübner \& Druey, 2006; Schuch \& Koch, 2004; Watter \& Logan, 2006), the use of finger-type categories should produce costs if the finger type repeats from the left hand $\left(T_{1}\right)$ to the right hand $\left(\mathrm{T}_{2}\right)$. An analogue relation also holds for the spatial categories. Thus, the use of spatial categories should produce costs if the relative button location (e.g., "left") repeats between hands (i.e., in $T_{1}$ and $T_{2}$ ).

For the present experiments it is crucial to notice that, with respect to the two abstract response category types (spatial, finger type), a repetition according to one category type (e.g., leftleft) coincides with a switch according to the other category type (e.g., middle finger-index finger; see Figure 2). Thus, in order to interpret eventual repetition effects, a certain perspective has to be adopted for analysing the data, since repetition costs according to one category type coincide with benefits according to the other category type. We therefore decided to analyse

\footnotetext{
${ }^{2}$ As can be seen in Figure 2, the anatomical finger features are completely equivalent to the spatial inner/outer features of the response buttons. In the following, though, we always differentiate between the spatial (in the sense of left/right) and the finger-type categories.
} 
Task switch

(Fixed task order and task-to-hand mapping)

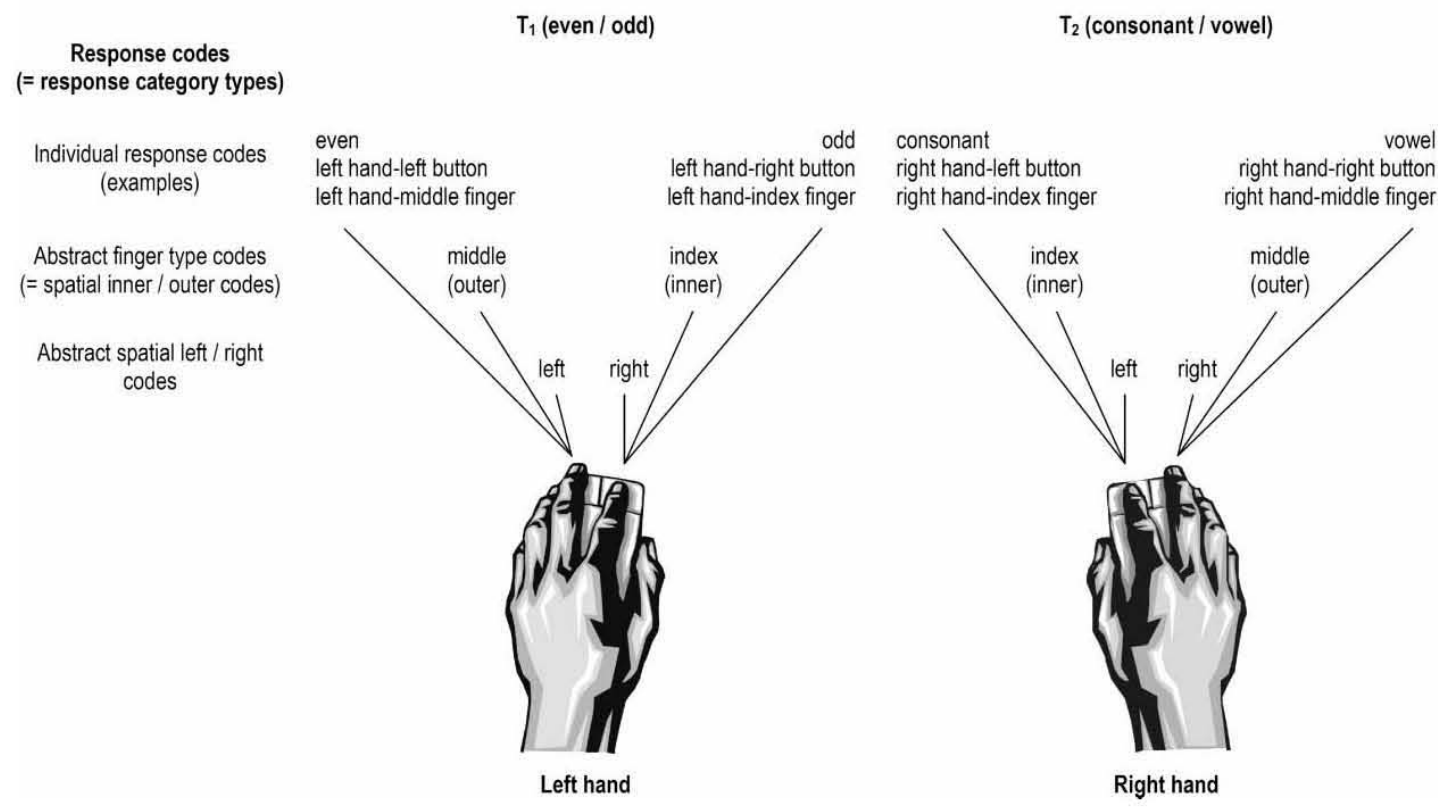

Figure 2. Possible response category types in a dual-task condition, in which task order and the task-to-hand mapping are fixed. Note that the responses can be coded individually either according to the corresponding stimulus features (e.g., in the sense of a response meaning; cf. Schuch $E^{\circ}$ Koch, 2004) or according to the combined and thus specific band-button or band-finger response features. Note further that the abstract finger-type response codes are equivalent to the spatial inner/outer response button position codes.

possible repetition effects always in terms of the spatial categories. Accordingly, eventual repetition costs would directly indicate that the participants used left/right as response codes. In contrast, repetition benefits would indicate that the responses were coded according to the finger-type features, since in previous studies repetition costs were usually observed under task switch conditions (Hübner \& Druey, 2006, in press; Schuch \& Koch, 2004).

\section{EXPERIMENT 1}

Our aim in the first experiment was to examine whether participants also use abstract response categories under conditions where the stimulus categories are mapped one-to-one to the responses. From several classification models (e.g., Gilbert \& Shallice, 2002; Logan \& Gordon, 2001;
Meiran, 2000a) as well as from the assumption that, in order to observe response category repetition effects, each task has to be performed with each hand (cf. Lien \& Proctor, 2002), one would not expect any repetition effects under such conditions. Nevertheless, it cannot a priori be excluded that abstract response categories are involved even under these conditions.

In this experiment, parity judgements for numerals and consonant/vowel judgements for letters served as tasks. As described in the Introduction, our task arrangement and the specific response layout (see Figure 2) allowed the participants to code their responses according to at least three different features. Which of these features was actually used for response coding should be revealed by the respective repetition effects.

Concerning the interpretation of a possible null effect, though, we have to be careful. As mentioned, if each response is coded individually 
(e.g., according to the individual effector), then there should be no repetition effects. The reverse conclusion, though, is not possible. If there are no repetition effects, then this does not necessarily indicate that the responses are coded individually. The reason is that the two other (abstract) response category types produce opposite effects. Consequently, if some participants use spatial categories, whereas others use finger-type categories, then we would not observe overall repetition effects, because the effects for the two groups counterbalance each other (cf. Stins \& Michaels, 1997, for a similar reasoning). Thus, an overall data analyses could result in an inappropriate conclusion regarding the involved response categories. Therefore, we also analysed the individual data.

\section{Method}

\section{Participants}

A total of 16 students ( 6 female, 10 male) participated in this experiment either for fulfilment of course requirements or for getting paid $5 € /$ hour. Their age ranged from 19 to 30 years $(M=22.25)$, and all reported normal or corrected-to-normal vision. All participants were right-handed by self report.

\section{Apparatus and stimuli}

Stimulus presentation and response recording were controlled by an IBM-compatible PC. The stimuli were presented on a $21^{\prime \prime}$ colour monitor with a resolution of $1,280 \times 768$ pixels and a refresh rate of $85 \mathrm{~Hz}$.

The digits 1, 2, 3, 4, 6, 7, 8, 9 and the letters $A$, E, I, U, G, K, M, R served as stimuli. They were presented in white on black background and subtended a visual angle of $2^{\circ}$ in height and approximately $1.36^{\circ}$ in width (depending on the individual stimulus) at a viewing distance of $110 \mathrm{~cm}$. The first stimulus $\left(\mathrm{S}_{1}\right)$ was always presented at the centre of the screen, whereas the second stimulus $\left(\mathrm{S}_{2}\right)$ appeared as (identical) flankers to the left and right of $\mathrm{S}_{1}$ at an eccentricity of approximately $1.18^{\circ}$ visual angle.

\section{Procedure}

The participants had to judge the parity (odd or even) of the numerals or the type (consonant or vowel) of the letters. Responses to $\mathrm{S}_{1}$ had to be given with the left hand and responses to $S_{2}$ with the right hand. For each hand there was an individual response box with two horizontally arranged response buttons. The task-to-hand mapping was always constant for a given participant throughout the whole experiment, but was balanced across participants. That is, half of the participants performed parity judgement as $T_{1}$ (i.e., with their left hand) and letter judgement as $T_{2}$ (i.e., with their right hand). Consequently, for these participants, digits always appeared as $\mathrm{S}_{1}$ and letters as $\mathrm{S}_{2}$. For the other half of the participants, task order was reversed. The categories "even" and "consonant" were mapped to the left response keys on each response box, and "odd" and "vowel" to the right response keys. These spatial features (as well as the finger types used for responding) were not, however, used for instructing the participants. Rather, by providing a similar depiction as in Figure 3, the individual buttons were designated by the stimulus features, with which they were associated throughout the experiment.

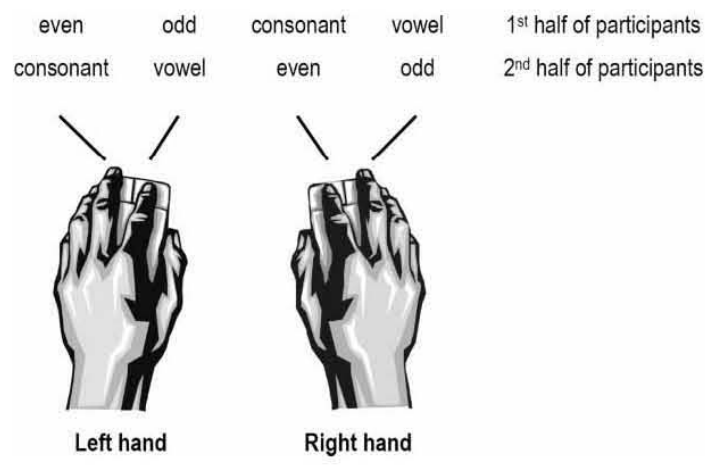

Figure 3. Depiction of the instruction of the two participant groups in Experiment 1. For the half of the participants who had to perform parity judgements as $T_{1}$ and letter judgements as $T_{2}$, a figure was used showing the upper line of $S R$-mappings. For the other balf of the participants who had to perform letter judgements as $T_{1}$ and digit judgements as $T_{2}$, a figure showing the lower line of $S R$-mappings was presented. 
A trial started with the presentation of a cue for $400 \mathrm{~ms}$ at the centre of the screen, indicating which judgement type was relevant for $\mathrm{S}_{1}$. The cue could have one of two forms: $\mathrm{g} / \mathrm{u}$ (abbreviations of the German words "gerade", even, and "ungerade", odd, indicating the parity judgement), and $\mathrm{k} / \mathrm{v}$ (abbreviations of the German words "Konsonant", consonant, and "Vokal", vowel, indicating the letter judgement). ${ }^{3}$ After cue presentation, a blank screen appeared for $600 \mathrm{~ms}$, followed by $S_{1} . S_{2}$ was then presented at a variable stimulus onset asynchrony (SOA) of 50, 150, 300, or $600 \mathrm{~ms}$ after $S_{1}$. Both $S_{1}$ and $S_{2}$ then remained on the screen until the participants responded. Immediately after the responding to $S_{2}$, the stimuli were replaced by a blank screen for $1,000 \mathrm{~ms}$ until presentation of the next cue. Participants were told to respond as fast and as accurately as possible first to $S_{1}$ and then also to $S_{2}$.

In total, the participants performed 18 blocks of 64 trials each in one 2 -hr session. The first two blocks were warm-up blocks and were not analysed.

\section{Results}

For the response times $\left(\mathrm{RT}_{1}\right.$ and $\mathrm{RT}_{2}$ in response to $S_{1}$ and $S_{2}$, respectively) as well as for the error rates, two-way repeated measures analyses of variance (ANOVAs) were computed, considering the factors $\operatorname{SOA}(50,150,300,600 \mathrm{~ms})$ and response (category repetition, category shift). As mentioned above, response category repetition was defined from the perspective of the spatial left/right dimension.

\section{$R_{1}$}

Response times. The analysis of $\mathrm{RT}_{1}$ revealed only a significant main effect of SOA, $F(3,45)=5.13$, $p<.01$. The response times at the individual SOAs from 50 to $600 \mathrm{~ms}$ were $717,698,685$, and $744 \mathrm{~ms}$, respectively.
Error rates. The analysis of the error rates in $\mathrm{R}_{1}$ revealed a significant main effect of response, $F(1,15)=13.01, p<.01$. If $\mathrm{S}_{1}$ and $\mathrm{S}_{2}$ required the same response category, then the participants made fewer errors $(1.84 \%)$ than when they required different response categories (3.71\%). Furthermore, the interaction between response and SOA was reliable, $F(3,45)=3.65, p<.05$, which reflects that the response correspondence effects decreased with increasing SOA (3.01, $2.12,0.37$, and $2.01 \%$ at the individual SOAs from 50 to $600 \mathrm{~ms}$ ).

$R_{2}$

Response times. For $\mathrm{RT}_{2}$, the analysis also revealed only a significant main effect of $\operatorname{SOA}, F(3,45)=$ 263.15, $p<.001$, reflecting a marked PRP effect (see Figure 4). The other effects were not significant.

Error rates. Regarding the error rates in $\mathrm{R}_{2}\left(\mathrm{ER}_{2}\right)$, the analysis revealed no significant effects. The mean $\mathrm{ER}_{2}$ was $4.59 \%$ (see Figure 4).

The results of the analyses considered so far seem to indicate that there were no response category repetition effects. However, this conclusion would only be justified if the averaged data pattern correctly reflects the individual patterns. Therefore, the data of the individual participants were inspected in order to determine which response categories they used. If the $\mathrm{RT}_{2}$ repetition effects for a given participant were in the same direction for at least three of the four SOAs, then this participant was assigned to the corresponding category group. ${ }^{4}$ In Figure 5, the $\mathrm{RT}_{2}$ data of two participants are exemplarily shown. As can be seen from this figure, for one participant (J.E., left graph) there were response category repetition costs, as one would expect if the responses had been coded in terms of left/ right. The other participant (T.L., right graph), though, shows response category repetition benefits. However, if one assumes that response

\footnotetext{
${ }^{3}$ Notice that the cue is completely redundant, since the task order is constant for a given participant throughout the whole experiment.

${ }^{4}$ The same criterion was also used for analysing the individual data in Experiments 2, 3, and 4.
} 


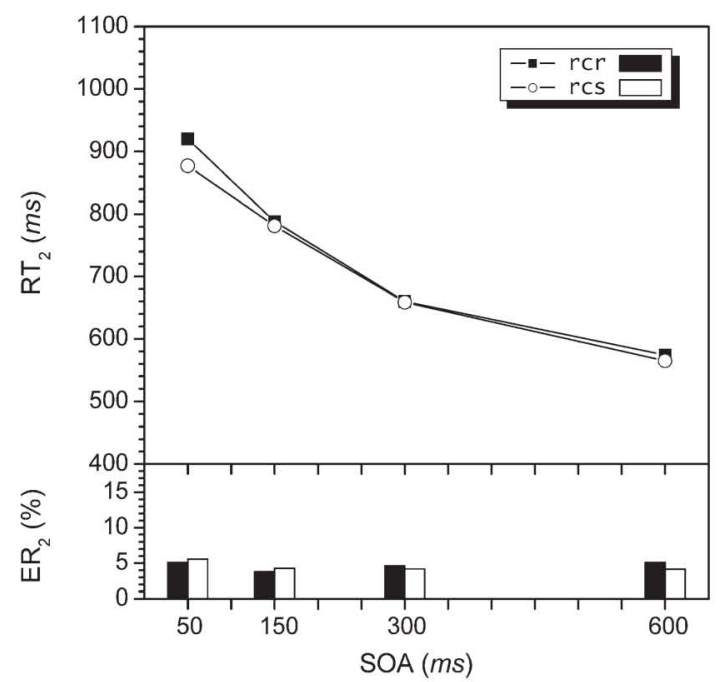

Figure 4. Overall latencies and error rates for $R_{2}$ in Experiment 1. The labels "rcr" and "rcs" in the legend denote "response category repetition" and "response category shift", respectively, according to the spatial left/right dimension.

category repetitions result in costs under task switching, then this rather suggests that T.L. used "index finger" and "middle finger" as response codes.

In total, this procedure thus resulted in two groups of unequal size: a total of 12 participants were assigned to the spatial left/right category group, and 4 participants were assigned to the

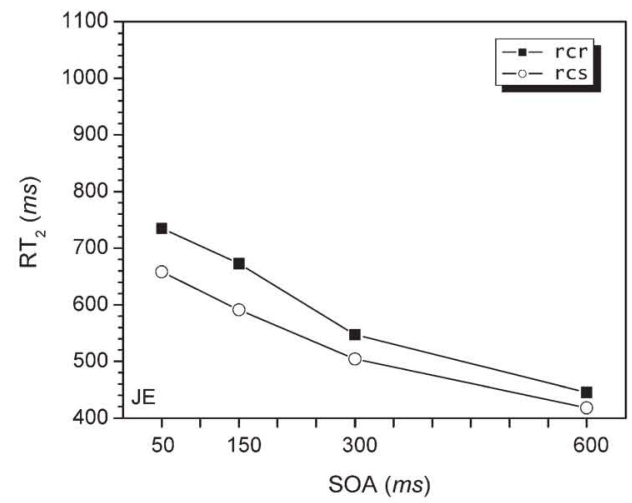

index/middle finger category group (averaged group data see Figure 6).

\section{Discussion}

Altogether, the results of the overall analysis first suggested that the participants did not code the responses according to one of the abstract response features, but rather individually (e.g., according to the individual effectors). However, from the inspection of the individual data patterns, it is obvious that, under the conditions established in this experiment, different participants nevertheless used different abstract types of response features as response codes. Whereas some participants seemed to rely on the finger features, the majority seemed to have used the spatial features. Thus, the present data are not in line with the assumption of Lien and Proctor (2002), according to which each task has to be performed with each hand in order to observe response category repetition effects. Furthermore, our results show that those categorization models, in which it is assumed that the responses are coded according to individual features (e.g., individual effectors or stimulus features, cf. Gilbert \& Shallice, 2002; Logan \& Gordon, 2001; Meiran, 2000a) are incomplete.

However, because our assignment of the participants to the respective groups was post hoc, we wanted to manipulate response coding directly.

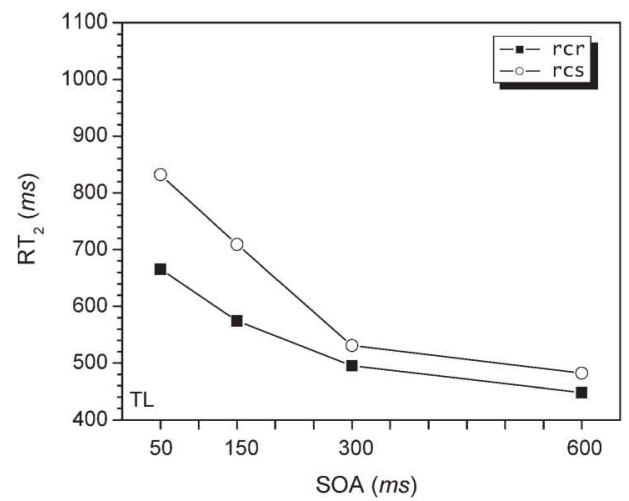

Figure 5. Two examples of individual RT data in Experiment 1. In the legend, "rcr" and "rcs" denote "response category repetition" and "response category shift", respectively, according to left/right. 


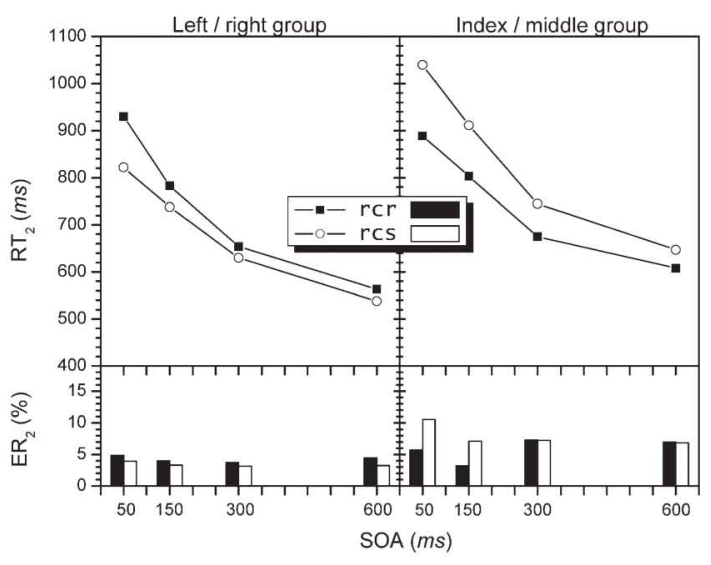

Figure 6. $R T_{2}$ and $E R_{2}$ in Experiment 1 for both category type groups. Left panel: Participants presumably applying left/right. Right panel: Participants presumably applying index/middle. The label " $r c r "=$ response category repetition; " $r c s "=$ response category shift; both according to left/ right.

Therefore, in the next experiment, the finger-type response codes were induced by training and instruction, since the individual data suggest that the application of these codes is less obvious than the application of the spatial codes.

\section{EXPERIMENT 2}

In this experiment, which consisted of two parts, the participants first went through alternating task switch and task repetition blocks (training session). In these training blocks, the participants had to perform colour and form judgements with traffic signs, and the stimulus categories (red, blue; round, angular) were mapped to specific finger types of both hands (i.e., index/middle finger). Furthermore, both judgement types served as $T_{1}$ and $T_{2}$, so that both tasks required responses with the respective fingers of both hands in the task repetition blocks as well as in the task switch blocks (i.e., variable task-to-hand mapping, see Figure 7). As mentioned in the Introduction, this results in invariance relations for the finger-type-based SR mappings across and within hands, which should encourage the

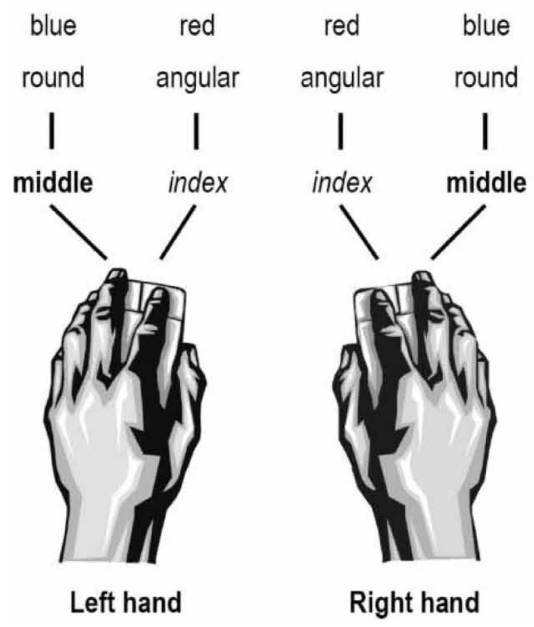

Figure 7. Stimulus-response mapping in the training blocks (task repetition and task switch) of Experiment 2.

participants to use these response features for response coding. In the second part of the experiment (test session), the participants performed the same task switch blocks with fixed task order and task-to-hand-mapping as in Experiment 1.

The reasoning behind applying such a two-step procedure with training and test session was to examine whether the participants maintained the response categories established in the training blocks also in the test blocks. Furthermore, we wanted to investigate whether the effects we observed in the previous experiment for the finger-type group were indeed due to coding the responses according to the finger-type response features. We expected that the participants would continue to use the already established response codes also in the test blocks, at least for some time, even though different stimuli and tasks are relevant in the two parts of the experiment. To further encourage the utilization of the finger-type response categories also in the test blocks, the stimulus categories were mapped to the individual fingers by instruction. According to Wenke and Frensch (2005), this should be sufficient for the participants to actually use the finger-type response codes even without prior training. Alternatively (or additionally), 
more participants than in Experiment 1 might code the responses according to the finger-type response features throughout the test blocks.

\section{Method}

A total of 16 students (11 female, 5 male) participated in the experiment under the same conditions as those in Experiment 1. Their age ranged from 19 to 38 years $(M=22.69)$.

The apparatus and the stimuli for the test blocks were the same as those in the previous experiment. In the additional training blocks, traffic signs (numbers 113, 140, 239, 245, 253, 255, 330, and 331 of the official German StVO sign catalogue) served as stimuli (see Figure 8).

\section{Procedure}

Training blocks. The participants had to categorize either the form (round or angular) or the colour (blue or red) of the presented traffic signs. As in our first experiment, responses to $S_{1}$ and $S_{2}$ had to be given with the index and middle fingers of

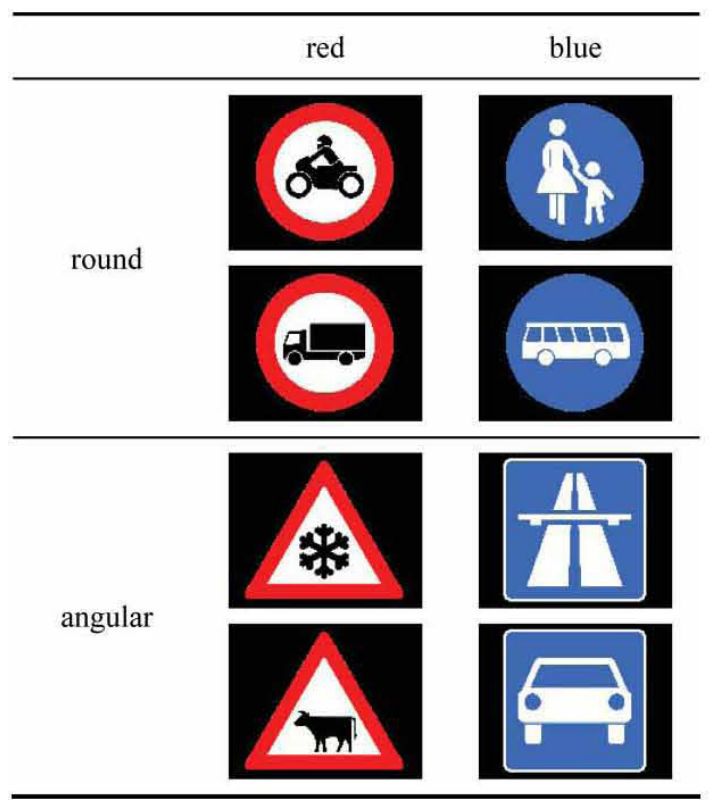

Figure 8. Depiction of the traffic sign stimuli applied in the training blocks of Experiment 2. the left and right hand, respectively. "Red" and "angular" were mapped to the index fingers of both hands, whereas "blue" and "round" were mapped to the middle fingers (see Figure 7). In total, the participants performed seven task repetition and seven task switch blocks with 64 trials each, which were presented in alternation, except for the first four blocks, where two taskswitch blocks followed two task-repetition blocks. These first four blocks served as warm-up blocks and were not analysed.

The temporal order of presentation on a given trial was the same as that in Experiment 1. "Form" (for the round/angular judgements) and "Farbe" (the German word for colour, for the red/blue judgements) served as task cues. As in the first experiment, the task cue always indicated the judgement type required for $\mathrm{S}_{1}$, and the judgement type for $\mathrm{S}_{2}$ was then defined by the required block type (cf. Hübner \& Druey, 2006). This resulted in a variable task order as well as in a variable task-to-hand mapping in the training blocks (e.g., form task as $T_{1}$ requiring a left-hand response, and colour task as $T_{2}$ requiring a righthand response on trial $n-1$; colour task as $T_{1}$ requiring a left-hand response, and form task as $\mathrm{T}_{2}$ requiring a right-hand response on trial $n$ ). Consequently, invariant SR mappings according to the index/middle finger response features were established in these blocks.

Test blocks. As test blocks, which immediately followed the training blocks, the participants had to perform four blocks of the same type as that in Experiment 1. However, the instructions were changed in that now the stimulus categories were mapped to finger types for both groups of participants. That is, for the first half of the participants, who always performed the parity task as $T_{1}$ and the letter task as $T_{2}$, "even" was (explicitly) mapped to the middle finger, and "odd" to the index finger of the left hand, whereas "consonant" was mapped to the index finger and "vowel" to the middle finger of the right hand. For the other half of the participants, who always performed the letter type task as $T_{1}$ and the parity task as $T_{2}$, the respective mappings were reversed. 


\section{Results}

\section{Training phase}

Since our focus was on the repetition effects in $R_{2}$, the latencies and error rates for $R_{1}$ were merely analysed by a one-way repeated measures ANOVA, considering the factor block type (task repetition, task switch). Regarding the $\mathrm{RT}_{2}$ and $\mathrm{ER}_{2}$ data, the factors block type (task repetition, task switch), SOA (50, 150, 300, $600 \mathrm{~ms})$, and response (category repetition, category shift) were considered in separate three-way repeated measures ANOVAs. Again, category repetition and category shift were considered according to the left/right response features (also in the figures).

$R_{1}$

Response times. For $\mathrm{RT}_{1}$, the analysis revealed a significant main effect of block type, $F(1,15)=$ $5.94, p<.05$. The participants responded faster in the task repetition blocks $(939 \mathrm{~ms}$ ) than in the task switch blocks (1,038 ms).

Error rates. Also for $\mathrm{ER}_{1}$, the analysis revealed a significant main effect of block type, $F(1,15)=$ $11.21, p<.01$. However, here the participants made more errors in the task repetition blocks $(6.75 \%)$ than in the task switch blocks (3.05\%).

$R_{2}$

Response times. The analysis of $\mathrm{RT}_{2}$ revealed significant main effects of block type, $F(1,15)=$ $43.17, p<.001$, and of SOA, $F(3,45)=161.72$, $p<.001$. The participants responded faster in the task repetition blocks than in the task switch blocks ( $965 \mathrm{~ms}$ vs. $1,391 \mathrm{~ms}$ ). The main effect of SOA reflects a reliable PRP effect. Furthermore, the interaction between block type and response was significant, $F(1,15)=56.57, p<.001$. As can be seen in Figure 9, leftmost panel, response category repetition resulted in "costs" of $130 \mathrm{~ms}$ in task repetition blocks, whereas it resulted in "benefits" of $149 \mathrm{~ms}$ in task switch blocks. Additionally, the two-way interaction between response and SOA, $F(3,45)=3.56, p<.05$, and the three-way interaction between block type, response, and SOA, $F(3,45)=5.92, p<.01$, were reliable (see also Figure 9, leftmost panel). These interactions are due to the fact that the repetition effects in the two block types showed partially opposite trends across the range of SOAs.

Error rates. The analysis of the $\mathrm{ER}_{2}$ data revealed a significant main effect of response, $F(1,15)=$ $30.16, p<.001$. The participants made more errors if the response category shifted (12.81\%) than if it repeated (6.94\%). Furthermore, there were two reliable two-way interactions. First, the interaction between response and block type was significant, $F(1,15)=53.60, p<.001$. In the task repetition blocks, there was no effect of response category repetition $(9.41 \%$ for category repetitions, $9.50 \%$ for category shifts). In the task switch blocks, however, the participants made considerably more errors if the response category shifted (16.21\%) than if it repeated (4.38\%). Second, the interaction of block type and SOA was also significant, $F(3,45)=3.16, p<.05$. Whereas the overall error rate increased slightly with increasing SOA in the task repetition blocks, it decreased in the task switch blocks. The error rates are also depicted in Figure 9 (leftmost panel).

\section{Test phase}

In order to examine whether the participants changed the response categories during the course of the test phase, the $R_{1}$ and $R_{2}$ data were entered into separate three-way repeated measures ANOVAs for the response times and error rates, considering the factors block $(1,2,3,4)$, response (category repetition, category shift), and $\mathrm{SOA}(50$, $150,300,600 \mathrm{~ms})$.

$R_{1}$

Response times. For $\mathrm{RT}_{1}$, there was a significant main effect of block, $F(1,15)=13.17, p<.01$. $\mathrm{RT}_{1}$ for the blocks 1 to 4 was $1,019,842,777$, and $733 \mathrm{~ms}$. For the factors response, $F(1,15)=$ $3.41, p=.085$, and SOA, $F(3,45)=2.62$, $p=.063$, the effects were marginally significant. 


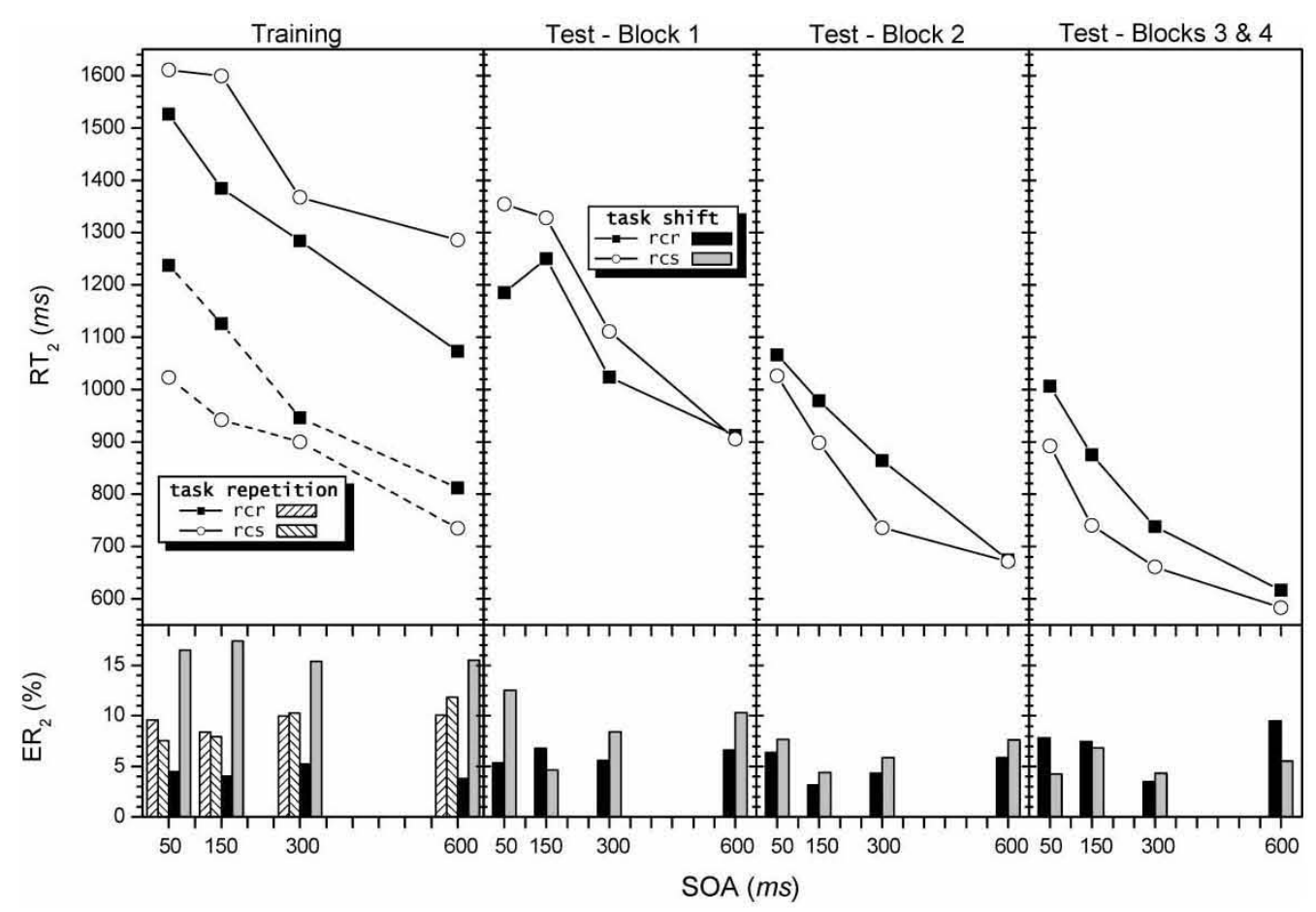

Figure 9. $R T_{2}$ and $E R_{2}$ in the training (leftmost panel) and test blocks of Experiment 2. Notice, that in the legend "rcr" and "res" denote "response category repetition" and "response category shift" according to the spatial dimension.

Whereas the response effect reflects a tendency towards slower responses with corresponding than with noncorresponding response categories in $T_{1}$ and $T_{2}$ ( $856 \mathrm{~ms}$ vs. $829 \mathrm{~ms}$, respectively), the SOA effect seems to be primarily due to slowed responses at the SOA of $600 \mathrm{~ms}$ ( $888 \mathrm{~ms}$ ), relative to the other SOAs from 50 to $300 \mathrm{~ms}$ (832, 824, and $827 \mathrm{~ms}$, respectively). Furthermore, the interaction of block and response was significant, $F(3,45)=3.52, p<.05$. This interaction reflects that, in the first block, corresponding response categories in $T_{1}$ and $T_{2}$ quickened responding by $50 \mathrm{~ms}$ relative to noncorresponding responses, whereas this pattern was reversed in the blocks 2 to 4 (correspondence costs of 31,87 , and $39 \mathrm{~ms}$, respectively).

Error rates. The analysis of $\mathrm{ER}_{1}$ revealed only a marginally significant main effect for block, $F(3$, $45)=2.43, p=.096$, reflecting decreasing error rates from Block 1 to Block 4 (5.28, 3.88, 2.73, and $2.31 \%$, respectively).

\section{$R_{2}$}

Response times. With respect to $\mathrm{RT}_{2}$, the analysis revealed a significant main effect of block, $F(3$, $45)=14.74, p<.001$, and of SOA, $F(3,45)=$ $100.05, p<.001$. Whereas the main effect of block reflects decreasing response times from Block 1 to Block $4(1,134,865,782$, and $745 \mathrm{~ms}$ ), the effect of SOA reflects a marked PRP-effect. The main effect of response was marginally significant, $F(1,15)=4.00, p=.064$, but was qualified by a reliable Block $\times$ Response interaction, $F(3,45)=6.24, p<.01$. As can be seen from Figure 9, there was a response category repetition "benefit" of $82 \mathrm{~ms}$ in the first block, which was marginally reliable, $F(1,15)=2.74, p=.059$ (one-tailed). In contrast, there were reliable costs 
of 61,110 , and $69 \mathrm{~ms}$ in Blocks 2 to $4, F_{\mathrm{s}}(1,15)>$ $4.03, p s<.05$ (one-tailed), respectively.

Error rates. Regarding $\mathrm{ER}_{2}$, there was a significant main effect of SOA, $F(3,45)=3.16, p<.05$. This effect reflects a U-shaped distribution of the error rates across the SOAs from 50 to $600 \mathrm{~ms}$ (7.01, 5.94, 4.97, and 7.56\%, respectively). Furthermore, the interaction of block and response reached marginal significance, $F(3,45)=2.57, p$ $=.066$, reflecting response category repetition "benefits" in Blocks 1 and 2 (2.89 and 1.46\%, respectively), and costs in Blocks 3 and 4 (1.17 and $2.51 \%$, respectively). These results are also depicted in Figure 9.

\section{Discussion}

As expected, by requiring task repetitions and by mapping the stimulus categories to certain finger types in the training phase, we were able to encourage response coding according to the finger types. This conclusion can be drawn from the respective repetition effects. Because response category repetitions were analysed with respect to left/right, we observed "costs" under task repetition, but "benefits" under task switching. Usually, these relations are reversed. However, this reversal is exactly what one would expect if the participants used the finger types as response codes. Generally, this supports our previous suggestion that participants prefer those categories, which produce invariant SR relations across and within hands (see also Hübner \& Druey, 2006, in press). Moreover, these results demonstrate that our training had the intended effects with respect to response coding. ${ }^{5}$

The crucial question was what happened in the test phase. Did the participants still use the finger types as response codes, as in the training phase? Since in the present experiment the participants received instructions according to the finger categories also in the test blocks, one could expect that this should be sufficient in order to induce the finger-type response codes also in these blocks (cf. Wenke \& Frensch, 2005), even if the tasks differed between training and test, and a fixed task-to-hand mapping was used in the test blocks.

As our data show, the participants indeed used the finger types as response codes, at least during the first test block. With respect to the $\mathrm{ER}_{2}$ repetition effects, the training (and instruction) effect was even present during the second test block (see Figure 9). Furthermore, the data for the first test block are similar to the task switch pattern in the training phase and to the pattern of repetition effects of the 4 participants in Experiment 1, for which we supposed finger-type response coding. However, it is also obvious from Figure 9 that, in the last two blocks, the participants switched to the spatial response categories, thus showing a pattern similar to that for the majority of the participants in Experiment 1 .

Also with respect to the individual results, the picture was quite clear. Whereas in the first test block 7 (out of the 16) participants showed a pattern of repetition effects indicating that they coded the responses according to the finger types, only 3 still showed a comparable pattern in Block 2 and none in Blocks 3 and 4 . With respect to the left/right response features, this pattern was reversed: Whereas only 5 participants showed a pattern indicating that they coded the responses according to the spatial features in Block 1 , already 7 used these features in Block 2 and 14 in Blocks 3 and 4. An unclear pattern was observed for 4 participants in Block 1, 6 participants in Block 2, and 2 participants in Blocks 3 and 4.

Altogether, the present results demonstrate that under the test conditions in Experiments 1 and 2, two abstract response category types (left/ right and index/middle finger) were used for response coding. Again, though, most participants

\footnotetext{
${ }^{5}$ With respect to the task switch effects, we observed the expected switch costs for $R_{2}$. Whereas the same holds also for $R_{1}$, if the response times are considered, task switch benefits occurred in the error rates. Thus, with respect to the task switch effects, we observed a speed-accuracy trade-off in the $\mathrm{R}_{1}$ results of the training blocks, which is not in line with our previous results (Hübner \& Druey, 2006).
} 
had a bias towards the spatial relative to the fingertype response categories. However, the mean as well as the individual data show that the transition from one category type to the other was not abrupt, but rather occurred at a different point in time for the different participants, respectively. Furthermore, the results in the second test block also support our view, that, even if several category types are simultaneously involved in response selection, nevertheless only one type is used for intentional SR translation and response inhibition (cf. Hübner \& Druey, in press). Thus, whereas in Test Block 2 the participants probably still relied on the finger-type response codes for SR translation and response inhibition on some trials, they may already have used the spatial codes on other trials (cf. Proctor et al., 2004, for a similar observation in a Simon task study).

Another interesting aspect of our data concerns the fact that we observed similar "repetition" (i.e., correspondence) costs in $\mathrm{RT}_{1}$ as in $\mathrm{RT}_{2}$. These correspondence costs in $\mathrm{RT}_{1}$ seem to be critical for our assumption of response inhibition as the core mechanism underlying the repetition costs in $\mathrm{R}_{2}$ on task switch trials. However, given the increasing response times with increasing SOA in $\mathrm{R}_{1}$, it might be quite simple to explain why correspondence costs occurred in $\mathrm{R}_{1}$ : Usually, if $\mathrm{RT}_{1}$ increases with increasing SOA under dual-task conditions, this is taken as evidence that the participants grouped their responses (e.g., Pashler \& Johnston, 1989). If this were the case here, then the correspondence costs in $\mathrm{RT}_{1}$ might simply reflect the already initiated inhibition of the respective response codes. This would especially be plausible given our previous result that response activation is sufficient for the inhibition process to be triggered, and that response execution is not necessary (Hübner \& Druey, 2006). The suspicion that response grouping accounts for the correspondence costs in $\mathrm{RT}_{1}$ is supported by an additional analysis of the $\mathrm{RT}_{1}$ data, in which we excluded trials with interresponse intervals of less than $150 \mathrm{~ms}$. Besides the fact that this eliminated the SOA effect $(F<1.5, p>.31)$, as expected, the response effect was no longer (marginally) significant $(F<2, p>.18)$. With respect to our conclusions about the response codes involved in response selection and inhibition, though, such a response grouping strategy is uncritical, since they are not based on the assumption of a specific mechanism producing the repetition effects in $R_{1}$ and $\mathrm{R}_{2}$.

The result that so many of the participants used the spatial response features for response coding despite instruction and training according to the finger features is nevertheless surprising. A possible reason for this preference of the spatial response codes could be that our stimuli also had spatial features. For both digits (e.g., Dehaene, Bossini, \& Giraux, 1993) and letters (Gevers, Reynvoet, \& Fias, 2003) it has been shown that they are mentally represented according to their spatial (left-to-right) features. Thus, there existed an overlap between the stimuli and the required responses with respect to this spatial dimension (cf. Kornblum et al., 1990). This could have caused a general tendency to also code the responses spatially (probably due to unspecific priming of the left/right codes). Therefore, the relevance of the stimulus features with respect to response coding was examined more thoroughly in the following experiments.

\section{EXPERIMENT 3}

In this experiment it should be examined whether the participants in the previous experiments coded the responses according to the left/right response features because the stimuli also had left/right features (cf. Dehaene et al., 1993; Gevers et al., 2003). As already mentioned in the Introduction, from Simon task studies it is well known that even task-irrelevant stimulus features affect task performance, if they overlap with corresponding response features. Thus, the spatial left/right stimulus features presumably not only automatically activated the corresponding response codes specifically, but rather also led to a general unspecific priming of this response code type relative to the finger-code type (cf. De Jong, Liang, \& Lauber, 1994; Hommel, 1998a; Kornblum et al., 1990; Ridderinkhof, 1997). 
In order to examine whether the spatial stimulus features were responsible for the "preference" of the spatial response codes in the previous experiments, the effects of spatial and nonspatial stimulus types (i.e., stimuli with and without implicit spatial features, respectively) were compared directly with respect to how they affect the response category repetition effects. In other words, we wanted to examine the extent to which stimulus features directly determine the type of response code involved in response selection and inhibition. Therefore, letters and digits served as stimuli in one condition and symbols and pictures, which obviously do not show spatial features overlapping with those of the responses, in another condition. ${ }^{6}$ Again, the participants performed only task-switch blocks, in which the tasks were uniquely linked to an individual hand for a given participant throughout the whole experiment. The different stimulus types and the corresponding tasks were blocked. All participants started with the letter and digit task blocks and then, in the second part of the experiment, shifted to the symbol and picture task blocks. As in the previous experiment, the instruction was according to the finger-type categories in both parts of the experiment. If the spatial features of the stimuli in the previous experiments were indeed responsible for the bias towards the spatial response categories, then, despite the instruction according to the finger types, the majority of the participants should code the responses according to the spatial categories in the first part of the experiment. In the second part, however, they might switch to the finger-type categories (cf. Wenke \& Frensch, 2005). Hence, the first part of the experiment also served as a control condition for the first and second experiments, since here only the instruction was according to the fingertype categories without prior training.

\section{Method}

\section{Participants and stimuli}

A total of 16 students (12 female, 4 male) participated in this experiment. Their age ranged from 19 to 31 years $(M=23.6)$. The apparatus, timing of presentation, and SOAs were the same as those in the previous experiments.

In the first part of the experiment, digits and letters as in the test blocks of Experiments 1 and 2 served as stimuli, and the corresponding tasks were parity and letter type judgements as before. In the second part of the experiment, symbols $(€, £, \S$, and $\&$ ) and pictures (dog, chipmunk, headphones, and pin) served as stimuli. With these stimuli, symbol (currency/noncurrency) and animal (animal/nonanimal) judgements had to be performed. The symbol and picture stimuli were also presented in white on black background and subtended visual angles of $1.58^{\circ} \pm 0.42^{\circ}$ in width and of $1.97^{\circ} \pm 0.33^{\circ}$ in height, depending on the individual stimulus.

\section{Procedure}

All participants started with digit and letter task blocks and then shifted to symbol and picture task blocks in the second part of the experiment. Task order within the two parts of the experiment was balanced across participants. For half of the participants, "odd" and "vowel" judgements required the middle and index fingers of the left hand, respectively, and "animal" and "currency" judgements required the index and middle fingers of the right hand, respectively. For the other half of the participants, "animal" and "currency" judgements required the middle and index fingers of the left hand, respectively, and "odd" and "vowel" judgements required the index and middle fingers of the right hand, respectively.

\footnotetext{
${ }^{6}$ Note that in the training blocks of Experiment 2 we already used stimuli (traffic signs) for which it can be assumed that they do not have implicit spatial features. Thus, despite the invariance relations, which resulted (a) from performing task repetition blocks and (b) from a variable task-to-hand mapping in the task switch blocks, this may have been a further reason why the participants followed the instructions and used the finger-type categories in these blocks. Nevertheless, the invariance relations seem to be more crucial in this respect, since the participants also code the responses according to the finger features with spatial stimuli (digits), if the mappings are accordingly (i.e., invariant, see e.g., Hübner \& Druey, in press; Watter \& Logan, 2006).
} 
Eight digit and letter task blocks were followed by eight symbol and picture task blocks. These blocks comprised 64 trials each, and all blocks were administered in one $2-\mathrm{hr}$ session.

\section{Results}

Separate three-way repeated measures ANOVAs with the factors response (category repetition, category shift), SOA (50, 150, 300, $600 \mathrm{~ms})$, and stimulus type (spatial, nonspatial) were computed for the $R_{1}$ and $R_{2}$ response times and error rates.

\section{$R_{1}$}

Response times. The analysis of $\mathrm{RT}_{1}$ revealed a significant main effect of $\mathrm{SOA}, F(3,45)=6.36$, $p<.01$. This effect is due to increased response times at the SOA of $600 \mathrm{~ms}(739 \mathrm{~ms})$ relative to the other SOAs $(729,727,726 \mathrm{~ms}$ at the individual SOAs from 50 to $300 \mathrm{~ms}$, respectively). However, this main effect was qualified by a marginally significant interaction between stimulus type and SOA, $F(3,45)=2.80, p=.051$. This interaction is due to the fact that for spatial stimuli (i.e., digits and letters), the response times to $\mathrm{S}_{1}$ showed a $\mathrm{U}$-shaped distribution across the SOA range $(734,728,730$, and $740 \mathrm{~ms}$ at the SOAs from 50 to $600 \mathrm{~ms}$, respectively), whereas for the nonspatial stimuli (i.e., symbols and pictures), the mean response times did not differ at the SOAs from 50 to $300 \mathrm{~ms}$ (723, 725, and $721 \mathrm{~ms}$, respectively), but the responses were substantially slower at the SOA of $600 \mathrm{~ms}$ (737 ms).

Error rates. Regarding $\mathrm{ER}_{1}$, only the interaction between SOA and response reached significance, $F(3,45)=3.55, p<.05$. This interaction reflects costs for response category repetitions at the SOAs of 50,150 , and $600 \mathrm{~ms}(0.13 \%, 0.17 \%$, and $0.23 \%$, respectively), but benefits at the SOA of $300 \mathrm{~ms}$ $(0.22 \%)$.

$R_{2}$

Response times. For $\mathrm{RT}_{2}$ there was a reliable main effect of SOA, $F(3,45)=358.34, p<.001$, reflecting the usual PRP-effect. This main effect, though, was qualified by a reliable two-way interaction between stimulus type and $\mathrm{SOA}, F(3,45)=$ $2.87, p<.05$ (see Figure 10). Most important, the interaction of response and stimulus type was significant, $F(1,15)=4.69, p<.05$. As can be seen in Figure 10, there were response category repetition costs for the digit and letter stimuli (29 ms), $F(1,15)=3.95, p<.05$, one-tailed, whereas for the symbol and picture stimuli there were response category repetition "benefits" $(20 \mathrm{~ms}), F(1,15)=7.90, p<.01$, one-tailed.

Error rates. Regarding $\mathrm{ER}_{2}$, only the three-way interaction between stimulus type, response, and SOA was significant, $F(3,45)=4.64, p<.01$. As can be seen in Figure 10, this interaction reflects that, for both parts of the experiment, the respective repetition effects at the SOA of $50 \mathrm{~ms}$ were reversed relative to the other SOAs.

\section{$R_{2}$ Part 1 (digit and letter stimuli)}

In order to examine whether the participants at least initially used the instructed finger-type categories with the digits and letters as stimuli, the response times and error rates of the first part were analysed separately in three-way repeated measures ANOVAs considering the factors block

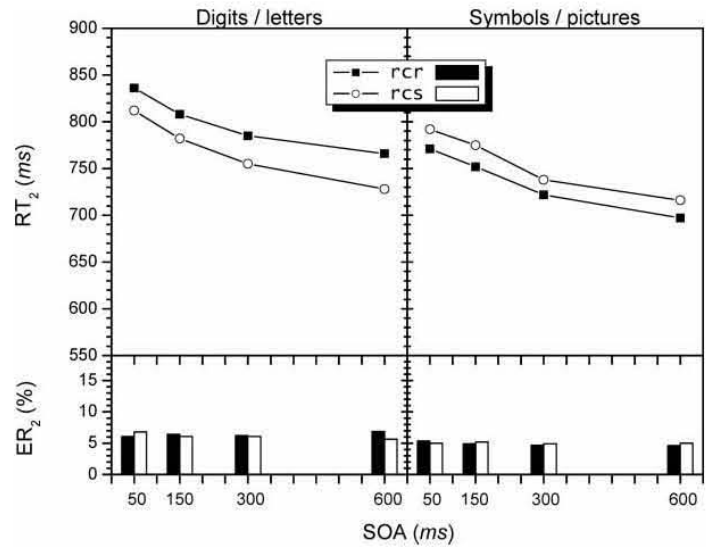

Figure 10. Overall $R T_{2}$ and $E R_{2}$ for both stimulus type parts (digits/letters, left panel; symbols/pictures, right panel) in Experiment 3. In the legend, "rr" denotes "response category repetition", and "rcs" denotes "response category shift", according to the spatial dimension. 
$(1,2,3,4,5,6,7,8)$, response (category repetition, category shift), and SOA $(50,150,300,600 \mathrm{~ms})$. For simplicity, only the reliable effects involving block are reported in the following.

Response times. For $\mathrm{RT}_{2}$, there was a significant main effect of block, $F(7,105)=15.60$, $p<.001$, reflecting a U-shaped distribution of the mean response times from Block 1 to Block $8(1,140,805,767,762,751,730,752$, and $753 \mathrm{~ms}$, respectively). Furthermore, the interaction of block and response reached significance, $F(7,105)=2.98, p<.01$. This interaction reflects that the repetition costs varied nonlinearly from Block 1 to Block $8(187,14,17,61,5,23$, $22,52 \mathrm{~ms}$, respectively)

Error rates. With respect to $\mathrm{ER}_{2}$, the analysis revealed no significant effect involving block.

\section{Discussion}

The results of this experiment show that different abstract response codes were used for the two stimulus-type conditions. Whereas costs occurred for the digits and letters, the symbols and pictures produced benefits for the repetition of the left/ right response categories between hands. These results suggest that response coding took place according to the left/right response features in blocks with digit and letter tasks, and according to the instructed finger types in blocks with symbol and picture tasks. The block-wise results further support this conclusion, since even in the first digit and letter task blocks the participants used left/right as response categories.

With respect to the individual results, the majority of the participants (9 out of 16) coded the responses according to the left/right response features in the digit and letter task blocks, as expected. Interestingly, though, 6 participants showed a pattern indicating that the instructed index/middle finger features were used as response categories in these blocks. One participant showed an unclear pattern of results in the first part. In the second part of the experiment (symbol and picture stimuli), 3 participants continued to use the left/right response categories, whereas 13 relied on the finger-type response codes.

However, although the results of the present experiment are largely as expected, there is also at least one critical aspect that should be considered: Due to not counterbalancing the order of the stimulus type conditions, there was a confound between stimulus type and practice. In the literature concerned with motor skill learning (e.g., Willingham, 1998), it has been shown that spatial (allocentric) motor codes become more and more effector-based (egocentric) with increasing practice. Although the participants did not really learn motor sequences here, it is still possible that this produced the respective repetition effects in the two parts of the present experiment. Therefore, in order to show that the present or absent spatial features of the stimuli were responsible for the different response codes with the different stimulus types, we had to rerun the experiment with a reversed stimulus type order.

This also allowed us to test a seeming discrepancy of the individual results in the present experiment and in Experiment 2: Whereas in the last two test blocks of Experiment 2 none of the participants used the finger-type response codes despite training and instruction according to this dimension, 6 participants relied on the fingertype codes throughout all digit and letter task blocks in the present experiment without preceding training. Probably this result simply reflects individual differences in the two examined samples. However, from the present data it is also possible that the training blocks in Experiment 2 produced this effect.

\section{EXPERIMENT 4}

In this experiment, all participants started with symbol and picture task blocks and then, in the second part of the experiment, shifted to the letter and digit task blocks. According to results of the previous experiments, we expected that the participants would follow the instruction and adopt the finger-type categories with the symbol and picture stimuli, since there should 
not be any stimulus-feature-driven priming of the spatial left/right response categories (cf. Wenke \& Frensch, 2005). Consequently, repetition costs for the finger-type categories should occur in this part of the experiment. However, if the spatial features of the presented digits and letters induce the left/right response codes, then the participants should shift from the fingertype response codes in the first part of the experiment to the spatial response codes in the second part. Thus, as in Experiment 3, we expected reversed repetition effects in the two parts of the experiment. Furthermore, with respect to the individual results, we expected a similar transition from one category type to the other as in Experiment 2.

If the effects in the previous experiment were due to a motor learning mechanism, though, which results in a transition from spatial to effector-based response coding with practice, then the effects should not differ between the two stimulus type parts of the present experiment-at least if the participants follow the instruction and code the responses according to the finger features in the first part with symbol and picture stimuli. However, given the results of Experiment 2, this seemed rather unlikely.

\section{Method}

\section{Participants, stimuli, and procedure}

A total of 16 students (12 female, 4 male) participated in this experiment. Their age ranged from 19 to 30 years $(M=22.7)$. The general method was the same as that in the previous experiment, with the exception that now the participants started with symbol and picture task blocks and then shifted to digit and letter task blocks in the second part of the experiment. Thus, eight symbol and picture task blocks were followed by eight digit and letter task blocks.

\section{Results}

Separate three-way repeated measures ANOVAs with the factors response (category repetition, category shift), $\operatorname{SOA}(50,150,300,600 \mathrm{~ms})$, and stimulus type (spatial, nonspatial) were computed for the $R_{1}$ and $R_{2}$ response times and error rates.

$R_{1}$

Response times. The analysis of $\mathrm{RT}_{1}$ revealed a significant main effect of $\mathrm{SOA}, F(3,45)=9.03$, $p<.001$. This effect is due to increased response times at the SOA of $600 \mathrm{~ms}(760 \mathrm{~ms})$ relative to the other SOAs (742, 741, $743 \mathrm{~ms}$ at the individual SOAs from 50 to $300 \mathrm{~ms}$, respectively). Also significant was the interaction between response and stimulus type, $F(1,15)=5.91, p<.05$. Whereas "benefits" occurred in the symbol and picture task blocks if $R_{1}$ and $R_{2}$ required the same response category $(8 \mathrm{~ms})$, there were costs in the digit and letter task blocks (19 ms). Finally, the interaction between response and SOA reached significance, $F(3,45)=3.77$, $p<.05$. The overall repetition effects (costs) varied in a nonlinear way across the SOA range.

Error rates. Regarding $\mathrm{ER}_{1}$, no effects were significant. On average, there were $4.28 \%$ errors.

$R_{2}$

Response times. For $\mathrm{RT}_{2}$ there was a reliable main effect of SOA, $F(3,45)=405.35, p<.001$, reflecting the usual PRP-effect. Most important, the interaction of response and stimulus type was significant, $F(1,15)=18.79, p<.001$. As can be seen in Figure 11, there were reliable response category repetition "benefits" for the symbol and picture stimuli $(42 \mathrm{~ms}), F(1,15)=11.53$, $p<.01$, one-tailed, whereas for the digit and letter stimuli there were reliable response category repetition costs $(24 \mathrm{~ms}), F(1,15)=3.27, p<.05$, one-tailed. Finally, the interaction between response and SOA was significant, $F(3,45)=$ $4.34, p<.01$, which is due to a nonlinear variation of repetition effects across the SOA range.

Error rates. Regarding $\mathrm{ER}_{2}$, there was only a significant main effect of $\mathrm{SOA}, F(3,45)=3.08$, $p<.05$, reflecting a $\mathrm{U}$-shaped distribution of error rates across the individual SOAs from 50 to $600 \mathrm{~ms}$ (see Figure 11). 




Figure 11. Overall $R T_{2}$ and $E R_{2}$ for both stimulus type parts (symbols/pictures, left panel; digits/letters, right panel) in Experiment 4. The label "ror" denotes "response category repetition", and "rcs" denotes "response category shift", according to the spatial dimension.

\section{$R_{2}$ Part 2 (digit and letter stimuli)}

In order to examine whether the participants continually switch the response categories throughout the test blocks as in Experiment 2, the response times and error rates of the second part (digit and letter stimuli) were analysed separately in three-way repeated measures ANOVAs considering the factors block $(1,2,3,4,5,6,7,8)$, response (category repetition, category shift), and SOA (50, $150,300,600 \mathrm{~ms})$. For simplicity, only the effects involving block are reported here.

Response times. For $\mathrm{RT}_{2}$, there was a significant main effect of block, $F(7,105)=8.91, p<.001$, reflecting a U-shaped distribution of the mean response times from Block 1 to Block 8 (1,085, $825,784,770,735,748,759$, and $795 \mathrm{~ms}$, respectively). However, the interaction of block and response was not significant $(F<1)$.

Error rates. With respect to $\mathrm{ER}_{2}$, the main effect of block was reliable, $F(7,105)=3.83, p<.001$. The frequency of errors decreased (although not strictly continually) from Block $1(10.57 \%)$ to Block 8
(4.52\%). Most important, though, the interaction of block and response reached marginal significance, $F(7,105)=1.94, p=.074$. Whereas there were response category repetition benefits between $6.03 \%$ and $1.29 \%$ in the first four blocks, costs between $0.43 \%$ and $2.24 \%$ occurred for the last four blocks (with the exception of Block 7).

\section{Discussion}

The results of the present experiment largely replicated the results of our previous experiments. Thus, as in Experiment 3, different abstract response codes were used under the two stimulus-type conditions. Whereas symbols and pictures produced benefits for the repetition of the left/right response categories between hands, costs occurred for digits and letters. These results suggest that response coding took place according to the instructed finger-type response features in blocks with symbol and picture tasks and according to the left/right response features in blocks with digit and letter tasks.

This conclusion receives further support from the individual data. As expected, the majority of the participants (13 out of 16) coded their responses according to the instructed index/ middle-finger-type response features, if symbols and pictures had to be categorized. Only 2 participants showed a pattern indicating that left/right response features were used for response coding in these blocks (and they kept this type of response code also for the digit and letter task blocks). One participant showed an unclear pattern of results in the first part. Of the 13 participants presumably using the finger-type response codes in the first part of the experiment, $5 \mathrm{kept}$ these codes also in the second part, whereas 7 switched to left/right response coding; 1 participant again showed an unclear pattern of results.

Compared with Experiment 2, the block-wise individual results were more heterogeneous. This may explain why the analysis of the transition from one type of response coding to the other revealed only a marginally significant effect in the error rates. Nevertheless, these results also 
show that different stimuli (with respect to their features) may induce different types of abstract response codes.

Taken together, the results of the present experiment also show that the transition from the spatial to the finger-type response codes in Experiment 3 was a consequence of the different features of the utilized stimuli, and not of motor learning (see, e.g., Willingham, 1998). This can be concluded from the fact that we observed similar effects in both experiments for the two stimulus type conditions: The participants used the finger-type response codes with symbols and pictures as stimuli and spatial response codes with digits and letters as stimuli. If motor skill learning would have been responsible for the transition from the spatial to the finger-type categories in Experiment 3, then we should have observed a similar transition in the present experiment. At least, the participants should have coded their responses according to the finger-type categories under both stimulus type conditions of the present experiment.

\section{GENERAL DISCUSSION}

The aim of the present study was to examine whether abstract response codes are involved in response selection and, if so, which types are used under conditions where at least two types (e.g., left/right or index/middle finger) are possible. Similar as in previous studies (e.g., Campbell \& Proctor, 1993), we analysed response category repetition effects to achieve this aim. Since we used a combined PRP and task-switch paradigm, where task order and task-to-hand mapping were fixed for each participant throughout the experiments, three different types of response codes were possible (see Figure 2). To determine which one of these codes was actually involved in response selection, we relied on the fact that response (category) repetitions usually produce costs under task switching (cf. Hübner \& Druey, 2006; Kleinsorge, 1999; Mayr \& Klieg1, 2003; Meiran, 2000a; Rogers \& Monsell,
1995; Schuch \& Koch, 2004; Watter \& Logan, 2006).

If no abstract response categories are involved, and the responses are coded individually (e.g., according to the individual effectors), then no repetition effects should arise because these codes cannot repeat from $T_{1}$ to $T_{2}$ (cf. Logan \& Schulkind, 2000). However, if the participants use one of the shared response features between hands for constructing abstract response categories (i.e., left/right or index/middle finger), then repetition costs should emerge in $\mathrm{R}_{2}$. Importantly, because the spatial and the finger-type mappings are crossed, the expected repetition effects are in opposite directions (cf. Proctor et al., 2004, for a similar procedure). That is, when viewed from the perspective of the spatial mapping, using the finger-type categories results in repetition benefits instead of costs.

The results of Experiment 1 illustrate all of these aspects. At first sight, the data seemed to indicate that the participants coded the responses according to individual features, since an overall analysis revealed no repetition effects. However, due to the fact that the spatial and the fingertype response codes produce opposite effects, the absence of an overall repetition effect cannot be viewed as clear evidence for this conclusion. It is also possible that some participants used fingertype codes whereas others used spatial codes, and that the opposite effects for these two types of response codes counterbalanced each other. Indeed, the analysis of the individual data indicated that this was the case. There were two groups of participants showing opposite repetition effects. From this result, two conclusions can be drawn: First, the responses were not coded in terms of individual and unique response features. Second, different participants used different response features as abstract response categories.

However, because we decided post hoc which participant used which type of response code, additional evidence was needed for the hypothesis that different participants prefer different types of response codes. Therefore, in Experiment 2, the participants were encouraged to code their responses according to the finger types by 
instruction and training under a certain SRmapping. As mentioned in the Introduction, invariant SR-relations across and within hands seem to be crucial for the coding of the responses according to a specific feature-category type. That is, if one wants the participants to code the responses according to the finger features, the stimulus categories should be mapped to finger types. Additionally, each hand should be required for responding to both tasks ( $\mathrm{cf}$. Lien \& Proctor, 2002). In a dual-task setting, these conditions are met if (a) also task repetitions have to be performed, and (b) in task-switch blocks, each judgement type serves as $T_{1}$ on some trials and as $T_{2}$ on others (i.e., task order is random but hand order is fixed). In the training blocks of Experiment 2, both of these conditions were realized.

As the results of the training blocks show, these measures had the intended effect. That is, the repetition effects that we observed indicated that the participants coded their responses according to the index/middle finger features in these blocks, as expected. The question now was whether they continued with this type of response code in the test blocks, which were identical to those in Experiment 1 (i.e., task switching only, with fixed task-to-hand mapping, digits and letters as stimuli). As the results of these blocks show, most of the participants did so, but only in the first test block. Thereafter, they shifted to the spatial codes. This raised the question of whether the preference for spatial response codes was due to a general dominance (of this category type over others) preference, or to some other specific aspect of our experimental context.

In order to answer this question, we examined the effects of the most obvious context factor, the stimuli. For both, digits (e.g., Bächtold, Baumüller, \& Brugger, 1998; Dehaene et al., 1993) and letters (Gevers et al., 2003), there is evidence that they have spatial features. Furthermore, from studies of the Simon effect (e.g., Simon, 1969; see Lu \& Proctor, 1995, for an overview), it is well known that overlapping features (or, to be more precise, overlapping feature representations) between the stimuli and the responses affect response selection (cf. e.g.,
Kornblum et al., 1990). In view of this evidence, the preference of the spatial over the finger-type response categories in our Experiments 1 and 2 might have been a consequence of using digits and letters as stimuli. The spatial features of these stimuli probably automatically activated (primed) the corresponding spatial categories.

In order to test this assumption, in Experiment 3 , the participants first performed blocks, in which letters and digits served as stimuli again. In a second part of the experiment, we then used symbol and picture stimuli, which had to be classified according to currency/noncurrency and animal/nonanimal, respectively. For the latter stimuli, we supposed that they are not mentally represented according to any implicit left-to-right ordered spatial features. In all other respects, the procedure was the same as that in the test blocks of Experiments 1 and 2. That is, only task switch blocks with fixed task order and task-to-hand mapping had to be performed. Furthermore, as in Experiment 2, the participants received instructions according to the finger-type response categories.

As expected, most of the participants followed the instruction and coded the responses according to the finger type in the blocks, in which symbols and pictures had to be categorized. This can be concluded from the overall repetition costs for the finger-type response categories. However, for the digit and letter stimuli in the first part of the experiment, we again observed overall repetition costs, indicating that, despite the instruction according to the finger-type categories, left/right served as response categories. Thus, the repetition effects in the two parts of Experiment 3 were in opposite directions, which may be taken as evidence that response selection (and, consequently, the inhibition of the respective response categories, cf. Hübner \& Druey, 2006; Steinhauser \& Hübner, 2006) involved different response category types in these two parts of the experiment. Furthermore, these results clearly show that digits and letters, at least in our tasks, automatically activate left/right response codes.

In Experiment 4, the order of the stimulus type blocks from our third experiment was reversed. Besides the fact that this experiment 
served as control and replication of the results of Experiment 3, it was also necessary for excluding the possibility that the transition from the spatial to the finger-type categories in the latter experiment was due to motor learning (cf. Willingham, 1998). As expected, the majority of the participants again coded the responses according to the finger features with the symbol and picture stimuli, but switched to the left/right response categories in the digit and letter task blocks. However, not all participants used the spatial categories for the digits and letters in the second part of the experiment, which indicates that the preceding experience with a finger mapping and different stimuli had a lasting effect. In all other aspects, the results of Experiment 4 largely replicated the results of Experiment 3. Thus, it is rather unlikely that the effects in Experiment 3 can be explained by motor learning, which usually results in a transition from spatial to effector-based movement representation with increasing practice.

In all experiments, letters (or words in the training blocks of Experiment 2) served as task cues. Accordingly, the cues also had implicit spatial features that might have primed the spatial response categories. Given the various results indicating that cues play a crucial role under task switching (e.g., priming of cue encoding and, consequently, memory retrieval of previous task transitions, Logan \& Schneider, 2006; Schneider \& Logan, 2006; task-set inhibition, Arbuthnott, 2005; Druey \& Hübner, in press), this aspect of our procedure could be problematic. Indeed, in Experiment 1, the specific cues could have encouraged the participants to use abstract spatial response categories. However, the results of Experiments 3 and 4 indicate that, if there is such an effect, it is much smaller than that of the stimuli, since the majority of the participants used the finger-type response codes for the nonspatial symbol and picture stimuli. Thus, altogether, the possible cue effects do not question our interpretation of the results.

From a general perspective, the observed effects are akin to those of stimulus affordance (e.g., Ellis \& Tucker, 2000; Tucker \& Ellis, 2001,
2004). Stimulus affordance means that object representations include not only feature descriptions, but also descriptions of actions that could be relevant for handling these objects (see also Hommel, 1998b; Hommel et al., 2001, and the concept of "event files"). Thus, stimulus affordance also implies that an object primes all the actions that are associated with it, including the currently inappropriate ones (Ellis \& Tucker, 2000; Tucker \& Ellis, 2004). Our results further support this idea.

\section{Homologous coupling as a bias factor towards spatial response coding?}

In studies of motor programming it has been shown that there is a tendency to coactivate homologous muscle groups on both sides of the body (i.e., homologous coupling, e.g., Heuer, 1993). Since we used the same fingers of both hands for responding to the required tasks, homologous coupling may have influenced our repetition effects especially at the short SOAs, where the programming stages for the two finger movements presumably overlapped temporally. Specifically, the repetition effects for the spatial response categories should have been increased, and the repetition effects for the finger-type categories should have been reduced if responding with homologous fingers is indeed fastened relative to responding with nonhomologous fingers. Because costs were expected for category repetitions under the conditions established in the present experiments and because we concluded from the repetition effects which category type the participants actually used, the potential coupling of homologous fingers would have biased our measure towards the detection of left/right response categories.

However, despite such a potential biasing effect of homologous coupling, the majority of participants showed substantial costs according to the finger-type categories in the blocks of Experiments 3 and 4, in which stimuli without implicit spatial features were used. Moreover, even with the spatial stimuli, there were at least some participants in the Experiments 1, 3, and 4 
who used the finger-type categories. Thus, the effects of homologous coupling, similar to the effects of cues with implicit spatial features, seem to be considerably smaller than the effects of the implicit spatial features of the stimuli, or the effects of the instruction.

\section{Response-effect or response-feature representation?}

Generally, there are two perspectives in the literature as to how responses in simple cognitive tasks are represented. On the one hand, several authors suppose that they are represented according to their intended and anticipated effects (e.g., Hommel et al., 2001; Kunde, 2001; Müsseler, Wühr, \& Prinz, 2000; Prinz, 1997). For instance, Müsseler et al. (2000) stated that "movements are cognitively represented by their external effects and can be initiated by the activation of these effect codes" (p. 745). On the other hand, there are conceptions according to which responses are primarily represented according to their features (e.g., Campbell \& Proctor, 1993; Hazeltine, 2005; Lu \& Proctor, 1995).

Our results fit better to the second perspective, since the sensory effects of pressing a given key were always the same. Thus, only if immanent response features are taken into account can the response category repetition effects observed for different participants and stimuli in our experiments be explained. However, this does not mean that response effects are irrelevant with respect to response coding (cf. e.g., Hommel, 1993). Rather, it suggests that response effects are not the only way according to which responses can be coded.

Moreover, action effects and response features cannot be distinguished in all conditions. Meiran (2000a), for instance, had participants judge the position of a target stimulus in a two-by-two grid according to the spatial categories either up/ down or left/right. The respective position had to be indicated by pressing one out of two response keys, which were located in diagonally opposite positions on a two-by-two response key box. Referring to Hommel (1997), he proposed a model in which the responses, once executed, are represented according to their respective effects. These effects, though, were defined in terms of the task-relevant spatial stimulus features. However, because the responses had the same features as the stimuli, response effects and response features cannot be distinguished in this case.

In any case, though, it is clear that, during the performance of a given task, the respective stimulus and response categories are associated. Such stimulus category - response category ( $\mathrm{SC}-\mathrm{RC}$ ) associations are supposed to play a crucial role with respect to the explanation of task switch costs (e.g., Meiran, Chorev, \& Sapir, 2000; Waszak, Hommel, \& Allport, 2003) and, according to some authors (e.g., Meiran, 2000a, 2000b; Schuch \& Koch, 2004), also with respect to the task sequence by response sequence interaction. Although we admit that the repetition costs that we observed in the present experiments may also be explained by the strengthening of task-relevant and the simultaneous weakening of task-irrelevant $\mathrm{SC}-\mathrm{RC}$ associations, the present data do not exclude alternative explanations. As already mentioned in the Introduction, given our previous results (Hübner \& Druey, 2006, in press; Steinhauser \& Hübner, 2006), we rather prefer an inhibition-based account.

\section{Costs with univalent stimuli?}

In the present study, we observed substantial costs for response category repetitions under task switching with univalent stimuli- that is, stimuli that were uniquely associated with only one task. This seems to be at odds with our previous study (Hübner \& Druey, 2006), where we observed such costs only for bivalent stimuli-that is, stimuli associated with both tasks - but not for univalent stimuli. This raises the question of why the observed repetition effects for univalent stimuli differ in both studies. One possible explanation could be that in our previous study there were also task repetition blocks, whereas in the present study only task switch blocks had to be performed. The additional experience with task repetitions might have led to a different overall strategy with respect to response inhibition, 
which is, according to our view, responsible for the repetition costs. Since strategies are presumably established quite early in an experiment, beginning with task repetition blocks might be critical in this respect. However, because we do not have independent evidence for this conjecture, future research has to show whether it is valid.

\section{CONCLUSIONS}

The results of the present experiments indicate that responses are coded in terms of abstract response-immanent features even in situations where the stimulus categories were unequivocally mapped to individual responses. Moreover, under the conditions realized in the present experiments, the participants used either the spatial features (i.e., left/right) of the response buttons or the anatomical finger features (i.e., index/middle finger) as abstract response codes. Finally, the participants usually rely on the response codes, according to which the SR-mapping is defined, but only if the features of the stimuli do not induce an alternative code-type.

\section{REFERENCES}

Arbuthnott, K. D. (2005). The influence of cue type on backward inhibition. Journal of Experimental Psychology: Learning, Memory, and Cognition, 31, 1030-1042.

Bächtold, D., Baumüller, M., \& Brugger, P. (1998). Stimulus response compatibility in representational space. Neuropsychologia, 26, 731-735.

Botvinick, M., Nystrom, L. E., Fissell, K., Carter, C. S., \& Cohen, J. D. (1999). Conflict monitoring versus selection-for-action in anterior cingulate cortex. Nature, 402, 179-181.

Campbell, K. C., \& Proctor, R. W. (1993). Repetition effects with categorizable stimulus and response sets. Journal of Experimental Psychology: Learning, Memory, and Cognition, 19, 1345-1362.
Cohen, A., \& Shoup, R. (1997). Perceptual dimensional constraints in response selection processes. Cognitive Psychology, 32, 128-181.

Dehaene, S., Bossini, S., \& Giraux, P. (1993). The mental representation of parity and number magnitude. Journal of Experimental Psychology: General, 122, 371-396.

De Jong, R., Liang, C. C., \& Lauber, E. (1994). Conditional and unconditional automaticity: A dualprocess model of effects of spatial stimulus-response correspondence. Journal of Experimental Psychology: Human Perception and Performance, 20, 731-750.

Druey, M. D., \& Hübner, R. (in press). The role of temporal cue-target overlap in backward inhibition under task switching. Psychonomic Bulletin $\dot{E}^{\circ}$ Review.

Duncan, J. (1979). Divided attention: The whole is more than the sum of its parts. Journal of Experimental Psychology: Human Perception and Performance, 5, 216-228.

Ellis, R., \& Tucker, M. (2000). Micro-affordance: The potentiation of components of action by seen objects. British Journal of Psychology, 91, 451-471.

Gevers, W., Reynvoet, B., \& Fias, W. (2003). The mental representation of ordinal sequences is spatially organized. Cognition, 87, B87-B95.

Gilbert, S. J., \& Shallice, T. (2002). Task switching: A PDP model. Cognitive Psychology, 44, 297-337.

Hazeltine, E. (2005). Response-response compatibility during bimanual movements: Evidence for the conceptual coding of action. Psychonomic Bulletin $\xi^{\circ}$ Review, 12, 682-688.

Hedge, A., \& Marsh, N. W. A. (1975). The effect of irrelevant spatial correspondences on two-choice response-time. Acta Psychologica, 39, 427-439.

Heister, G., Schroeder-Heister, P., \& Ehrenstein, W. H. (1990). Spatial coding and spatio-anatomical mapping: Evidence for a hierarchical model of spatial stimulus-response compatibility. In R. W. Proctor \& T. G. Reeve (Eds.), Stimulus-response compatibility (pp. 117-143). Amsterdam: North-Holland.

Heuer, H. (1993). Structural constraints on bimanual movements. Psychological Research, 55, 83-98.

Hommel, B. (1993). Inverting the Simon effect by intention: Determinants of direction and extent of effects of irrelevant spatial information. Psychological Research, 55, 270-279.

Hommel, B. (1997). Toward an action-concept model of stimulus-response compatibility. In B. Hommel \& W. Prinz (Eds.), Theoretical issues in stimulusresponse compatibility (pp. 281-320). Amsterdam: North-Holland.

\section{8}


Hommel, B. (1998a). Automatic stimulus-response translation in dual-task performance. Joumal of Experimental Psychology: Human Perception and Performance, 24, 1368-1384.

Hommel, B. (1998b). Event files: Evidence for automatic integration of stimulus-response episodes. Visual Cognition, 5, 183-216.

Hommel, B., Müsseler, J., Aschersleben, G., \& Prinz, W. (2001). The theory of event coding (TEC): A framework for perception and action planning. Behavioral and Brain Sciences, 24, 849-937.

Houghton, G., \& Tipper, S. P. (1994). A model of inhibitory mechanisms in selective attention. In D. Dagenbach \& T. H. Carr (Eds.), Inbibitory processes in attention, memory, and language (pp. 53-112). San Diego, CA: Academic Press.

Hübner, R., \& Druey, M. D. (2006). Response execution, selection, or activation: What is sufficient for response-related repetition effects under task shifting? Psychological Research, 70, 245-261.

Hübner, R., \& Druey, M. D. (in press). Multiple response codes play specific roles in response selection and inhibition under task shifting. Psychological Research.

Kleinsorge, T. (1999). Response repetition benefits and costs. Acta Psychologica, 103, 295-310.

Kornblum, S., Hasbroucq, T., \& Osman, A. (1990). Dimensional overlap: Cognitive basis for stimulusresponse compatibility - a model and taxonomy. Psychological Review, 97, 253-270.

Kornblum, S., \& Lee, J. W. (1995). Stimulus-response compatibility with relevant and irrelevant stimulus dimensions that do and do not overlap with the response. Journal of Experimental Psychology: Human Perception and Performance, 21, 855-875.

Kunde, W. (2001). Response-effect compatibility in manual choice reaction tasks. Journal of Experimental Psychology: Human Perception and Performance, 27, 387-394.

Lamberts, K. (2000). Information-accumulation theory of speeded categorization. Psychological Review, $107,227-260$.

Lien, M. C., \& Proctor, R. W. (2000). Multiple spatial correspondence effects on dual-task performance. Journal of Experimental Psychology: Human Perception and Performance, 26, 1260-1280.

Lien, M. C., \& Proctor, R. W. (2002). Stimulusresponse compatibility and psychological refractory period effects: Implications for response selection. Psychonomic Bulletin E Review, 9, 212-238.
Lien, M. C., Schweickert, R., \& Proctor, R. W. (2003). Task switching and response correspondence in the psychological refractory period paradigm. Joumal of Experimental Psychology: Human Perception and Performance, 29, 692-712.

Logan, G. D. (1988). Toward an instance theory of automatization. Psychological Review, 95, 492-527.

Logan, G. D. (2002). An instance theory of attention and memory. Psychological Review, 109, 376-400.

Logan, G. D., \& Gordon, R. D. (2001). Executive control of visual attention in dual-task situations. Psychological Review, 108, 393-434.

Logan, G. D., \& Schneider, D. W. (2006). Priming or executive control? Associative priming of cue encoding increases "switch costs" in the explicit task-cuing procedure. Memory E Cognition, 34, 1250-1259.

Logan, G. D., \& Schulkind, M. D. (2000). Parallel memory retrieval in dual-task situations: I. Semantic memory. Journal of Experimental Psychology: Human Perception and Performance, 26, 1072-1090.

Lu, C. H., \& Proctor, R. W. (1995). The influence of irrelevant location information on performance: A review of the Simon and spatial Stroop effects. Psychonomic Bulletin Eं Review, 2, 174-207.

Mayr, U., \& Kliegl, R. (2003). Differential effects of cue changes and task changes on task-set selection costs. Journal of Experimental Psychology: Leaming, Memory, and Cognition, 29, 362-372.

Meiran, N. (2000a). Modeling cognitive control in taskswitching. Psychological Research, 63, 234-249.

Meiran, N. (2000b). Reconfiguration of stimulus task sets and response task sets during task switching. In S. Monsell \& J. Driver (Eds.), Attention and performance XVIII: Control of cognitive processes (pp. 377-399). Cambridge, MA: MIT Press.

Meiran, N., Chorev, Z., \& Sapir, A. (2000). Component processes in task switching. Cognitive Psychology, 41, 211-253.

Müsseler, J., Wühr, P., \& Prinz, W. (2000). Varying the response code in the blindness to response-compatible stimuli. Visual Cognition, 7, 743-767.

Nosofsky, R. M., \& Palmeri, T. J. (1997). An exemplarbased random walk model of speeded classification. Psychological Review, 104, 266-300.

Pashler, H., \& Baylis, G. (1991). Procedural learning: 2. Intertrial repetition effects in speeded-choice tasks. Journal of Experimental Psychology: Learning, Memory, and Cognition, 17, 33-48. 